\title{
An application of the Gaussian correlation inequality to the small deviations for a Kolmogorov diffusion*
}

\author{
Marco Carfagnini ${ }^{\dagger}$
}

\begin{abstract}
We consider an iterated Kolmogorov diffusion $X_{t}$ of step $n$. The small ball problem for $X_{t}$ is solved by means of the Gaussian correlation inequality. We also prove Chung's laws of iterated logarithm for $X_{t}$ both at time zero and infinity.
\end{abstract}

Keywords: Diffusion processes, Kolmogorov diffusion, functional limit laws, small ball problem. MSC2020 subject classifications: Primary 60F17, Secondary 60F15; 60G51; 60J65.

Submitted to ECP on January 2, 2022, final version accepted on February 22, 2022.

\section{Contents}

1 Introduction

2 The setting and main results

References

\section{Introduction}

Let $\left\{X_{t}\right\}_{0 \leqslant t \leqslant T}$ be an $\mathbb{R}^{n}$-valued stochastic process with continuous paths such that $X_{0}=0$ a.s. where $T>0$ is fixed. Denote by $W_{0}\left(\mathbb{R}^{n}\right)$ the space of $\mathbb{R}^{n}$-valued continuous functions on $[0, T]$ starting at zero. Given a norm $\|\cdot\|$ on $W_{0}\left(\mathbb{R}^{n}\right)$, the small ball problem for $X_{t}$ consists in finding the rate of explosion of

$$
-\log \mathbb{P}(\|X\|<\varepsilon)
$$

as $\varepsilon \rightarrow 0$. More precisely, a process $X_{t}$ is said to satisfy a small deviation principle with rates $\alpha$ and $\beta$ if there exist a constant $c>0$ such that

$$
\lim _{\varepsilon \rightarrow 0}-\varepsilon^{\alpha}|\log \varepsilon|^{\beta} \log \mathbb{P}(\|X\|<\varepsilon)=c .
$$

The values of $\alpha, \beta$ and $c$ depend on the process $X_{t}$ and on the chosen norm on $W_{0}\left(\mathbb{R}^{n}\right)$. Small deviation principles have many applications including metric entropy estimates

\footnotetext{
*Research was supported in part by NSF Grants DMS-1712427 and DMS-1954264.

${ }^{\dagger}$ University of Connecticut, United States of America. E-mail: marco. carfagnini@uconn . edu
} 
and Chung's law of the iterated logarithm. We refer to the survey paper [13] for more details.

We say that a process $X_{t}$ satisfies Chung's law of the iterated logarithm (LIL) as $t \rightarrow \infty$ (resp. as $t \rightarrow 0$ ) with rate $a \in \mathbb{R}_{+}$if there exists a constant $C$ such that

$$
\liminf _{t \rightarrow \infty}\left(\frac{\log \log t}{t}\right)^{a} \max _{0 \leqslant s \leqslant t}\left|X_{s}\right|=C, \quad \text { a.s. }
$$

(resp. $\liminf \inf _{t \rightarrow 0}\left(\frac{\log |\log t|}{t}\right)^{a} \max _{0 \leqslant s \leqslant t}\left|X_{s}\right|=C$ a.s.). When $X_{t}$ is a Brownian motion, it was proven in a famous paper by K.-L. Chung in 1948 that (1.2) holds with $a=\frac{1}{2}$ and $C=\frac{\pi}{\sqrt{8}}$. To find the rates $\alpha$ and $\beta$ such that the limit in (1.1) exists, and then findind the constant $c$ is an extremely hard problem in general. Even the estimation of the rate of explosion of (1.1) is usually a difficult problem. Indeed, as can be surmised in [10, 15], the small ball problem for Gaussian processes is equivalent to metric entropy problems in functional analysis. In [11] and [20] a Brownian sheet in Hölder and uniform norm is considered, and the integrated Brownian motion in the uniform norm is the content of [8], and the m-fold integrated Brownian motion in both the uniform and $L^{2}$-norm is considered in [4]. In [17] and [3] a small deviation principle and Chung's LIL are proved for a class of stochastic integrals and for a hypoelliptic Brownian motion on the Heisenberg group. When $X_{t}$ is a Gaussian process with stationary increments, upper and lower bounds on (1.1) can be found in [19, 16].

In this paper we consider the Kolmogorov diffusion of step $n$.

Definition 1.1. Let $T>0$ and $b_{t}$ be a one-dimensional standard Brownian motion. The stochastic process $\left\{X_{t}\right\}_{0 \leqslant t \leqslant T}$ on $\mathbb{R}^{n}$ defined by

$$
X_{t}:=\left(b_{t}, \int_{0}^{t} b_{t_{2}} d t_{2}, \int_{0}^{t} \int_{0}^{t_{2}} b_{t_{3}} d t_{3} d t_{2}, \ldots, \int_{0}^{t} \int_{0}^{t_{2}} \ldots \int_{0}^{t_{n-1}} b_{t_{n}} d t_{n} \ldots d t_{2}\right)
$$

is the Kolmogorov diffusion of step $n$.

$\left\{X_{t}\right\}_{0 \leqslant t \leqslant T}$ is a Markov process with generator given by $L=\frac{1}{2} \frac{\partial^{2}}{\partial x_{1}^{2}}+\sum_{d=2}^{n} x_{d-1} \frac{\partial}{\partial x_{d}}$. In particular, when $n=2 X_{t}$ is the Markov process associated to the differential operator $L=\frac{1}{2} \frac{\partial^{2}}{\partial x^{2}}+x \frac{\partial}{\partial y}$ and it was first introduced by A. N. Kolmogorov in [9], where he obtained an explicit expression for its transition density. Later, L. Hörmander in [6] used $L$ as the simplest example of a hypoelliptic second order differential operator. More precisely, the operator $L$ satisfies the weak Hörmander condition. $\left\{X_{t}\right\}_{0 \leqslant t \leqslant T}$ is a Gaussian process and its law $\mu$ is a Gaussian measure on the Banach space $\left(W_{0}\left(\mathbb{R}^{n}\right),\|\cdot\|\right)$, where

$$
\|f\|:=\max _{0 \leqslant t \leqslant T}|f(t)|, \quad \forall f \in W_{0}\left(\mathbb{R}^{n}\right) .
$$

The main result of this paper is Theorem 2.6, where we prove the small deviation principle (1.1) for $X_{t}$ with rates $\alpha=2, \beta=0$, and constant $c=\frac{\pi}{\sqrt{8}}$. Our proof relies on the Gaussian correlation inequality (GCI), see e.g. $[18,12]$, applied to the Gaussian measure $\mu$ on $W_{0}\left(\mathbb{R}^{n}\right)$. A different application of the GCI to estimate small balls probabilities is given in [14]. In Theorem 2.6 we also state Chung's LIL at time zero and infinity for $X_{t}$ with rates given by $a=\frac{1}{2}$ and $a=\frac{2 n-1}{2}$ respectively.

The stochastic processes considered in $[8,17,3]$ all satisfy a scaling property, that is, there exists a scaling constant $\delta \in(0, \infty)$ such that $X_{\varepsilon t} \stackrel{(d)}{=} \varepsilon^{\delta} X_{t}$. Properties of Gaussian measures on Banach spaces and scaling properties have been used to show the existence of a small deviation principle for some processes such as a Brownian motion with values in a finite dimensional Banach space in [5] and an integrated Brownian motion in [8]. Moreover, in $[8,17,3]$ the scaling rate $\delta$ coincides with the rate of Chung's LIL at infinity, 
and the small deviations' rates are given by $\beta=0, \alpha=\frac{1}{\delta}$. The Kolmogorov diffusion does not satisfy a scaling property with respect to the standard Euclidean norm, and the small deviations rate $\alpha$ is not related to the Chung's LIL rate.

Lastly, large deviations and Chung's LIL at time zero for the limsup of the Kolmogorov diffusion are discussed in Section [1, Section 4.2] and [2, Example 3.5] respectively.

The paper is organized as follows. In Section 2 we collect some examples and state the main result of this paper, namely, small deviation principle and Chung's LIL at time zero and infinity for a step $n$ Kolmogorov diffusion. Section 3 contains the proof of the main result.

\section{The setting and main results}

Notation 2.1. 4 Let $X_{t}$ be an $\mathbb{R}^{n}$-valued stochastic process with $X_{0}=0$ a.s. Then $X_{t}^{*}$ denotes the process defined by

$$
X_{t}^{*}:=\max _{0 \leqslant s \leqslant t}\left|X_{s}\right|,
$$

where $|\cdot|$ denotes the Euclidean norm.

Notation 2.2. [Dirichlet eigenvalues in $\mathbb{R}^{n}$ ] We denote by $\lambda_{1}^{(n)}$ the lowest Dirichlet eigenvalue of $-\frac{1}{2} \Delta_{\mathbb{R}^{n}}$ on the unit ball in $\mathbb{R}^{n}$.

Let us collect some examples of Chung's LIL and small deviation principle.

Example 2.3. [Brownian motion] Let $X_{t}$ be a standard Brownian motion. Then $X_{\varepsilon t} \stackrel{(d)}{=}$ $\varepsilon^{\frac{1}{2}} X_{t}$, and it satisfies the small deviation principle

$$
\lim _{\varepsilon \rightarrow 0}-\varepsilon^{2} \log \mathbb{P}\left(X_{T}^{*}<\varepsilon\right)=\lambda_{1}^{(1)} T,
$$

where $\lambda_{1}^{(1)}$ is defined in Notation 2.2, see e.g. [7, Lemma 8.1]. Moreover, in a famous paper by K.-L. Chung in 1948 it was proven that

$$
\liminf _{t \rightarrow \infty}\left(\frac{\log \log t}{t}\right)^{\frac{1}{2}} \max _{0 \leqslant s \leqslant t}\left|X_{t}\right|=\sqrt{\lambda_{1}^{(1)}} \text { a.s. }
$$

Example 2.4. [Integrated Brownian motion]. Let $X_{t}:=\int_{0}^{t} b_{s} d s$, where $b_{s}$ is a onedimensional standard Brownian motion. It is easy to see that $X_{\varepsilon t} \stackrel{(d)}{=} \varepsilon^{\frac{3}{2}} X_{t}$. In [8] it is shown that there exists a finite constant $c_{0}>0$ such that

$$
\liminf _{t \rightarrow \infty}\left(\frac{\log \log t}{t}\right)^{\frac{3}{2}} \max _{0 \leqslant s \leqslant t}\left|X_{t}\right|=c_{0} \quad \text { a.s. }
$$

and (2.3) was used to prove that

$$
\lim _{\varepsilon \rightarrow 0}-\varepsilon^{\frac{2}{3}} \log \mathbb{P}\left(X_{1}^{*}<\varepsilon\right)=c_{0}^{\frac{2}{3}} .
$$

Example 2.5. [Iterated integrated Brownian motion] Let $b_{t}$ be a one-dimensional Brownian motion starting at zero. Denote by $X_{1}(t):=b_{t}$ and

$$
X_{d}(t):=\int_{0}^{t} X_{d-1}(s) d s, \quad t \geqslant 0, d \geqslant 2
$$

the $d$-fold integrated Brownian motion for a positive integer $d$. Note that $X_{d}(\varepsilon t) \stackrel{(d)}{=}$ $\varepsilon^{\frac{2 d-1}{2}} X_{d}(t)$. In [4] it was shown that for any integer $d$ there exists a constant $\gamma_{d}>0$ such 
that

$$
\begin{aligned}
& \lim _{\varepsilon \rightarrow 0}-\varepsilon^{\frac{2}{2 d-1}} \log \mathbb{P}\left(\max _{0 \leqslant t \leqslant 1}\left|X_{d}(t)\right|<\varepsilon\right)=\gamma_{d}^{\frac{2}{2 d-1}}, \\
& \liminf _{t \rightarrow \infty}\left(\frac{\log \log t}{t}\right)^{\frac{2 d-1}{2}} \max _{0 \leqslant s \leqslant t}\left|X_{d}(s)\right|=\gamma_{d} \text { a.s. }
\end{aligned}
$$

Our main object is the Kolmogorov diffusion on $\mathbb{R}^{n}$ defined by

$$
X_{t}:=\left(X_{1}(t), \ldots, X_{n}(t)\right)
$$

where

$$
X_{d}(t):=\int_{0}^{t} \int_{0}^{t_{2}} \cdots \int_{0}^{t_{d-1}} b_{t_{d}} d t_{d} \cdots d t_{2}, \text { for } d=3, \ldots, n,
$$

and $X_{2}(t):=\int_{0}^{t} b_{s} d s, X_{1}(t):=b_{t}$, where $b_{t}$ is a one-dimensional standard Brownian motion. Note that $X_{d}(\varepsilon t) \stackrel{(d)}{=} \varepsilon^{\frac{2 d-1}{2}} X_{d}(t)$ for all $d=1, \ldots, n$, and hence the process $X_{t}$ does not have a scaling property with respect to the Euclidean norm $|\cdot|$ in $\mathbb{R}^{n}$.

Theorem 2.6. Let $T>0$ and $X_{t}$ be the Kolmogorov diffusion on $\mathbb{R}^{n}$. Then

$$
\begin{aligned}
& \lim _{\varepsilon \rightarrow 0}-\varepsilon^{2} \log \mathbb{P}\left(X_{T}^{*}<\varepsilon\right)=\lambda_{1}^{(1)} T, \\
& \liminf _{t \rightarrow 0} \sqrt{\frac{\log |\log t|}{t}} \max _{0 \leqslant s \leqslant t}\left|X_{s}\right|=\sqrt{\lambda_{1}^{(1)}} \quad \text { a.s. } \\
& \liminf _{t \rightarrow \infty}\left(\frac{\log \log t}{t}\right)^{\frac{2 n-1}{2}} \max _{0 \leqslant s \leqslant t}\left|X_{s}\right|=\gamma_{n} \quad \text { a.s. }
\end{aligned}
$$

where $\lambda_{1}^{(1)}$ is defined in Notation 2.2, and $\gamma_{n}$ is given by (2.4)

Remark 2.7. By (2.2) and Brownian inversion, it follows that a standard Brownian motion satisfies Chung's LIL at time zero and infinity with rate $a=\frac{1}{2}$, and it satisfies a small deviation principle with rate $\alpha=2$. By (2.5), the $n$-step Kolmogorov diffusion $X_{t}$ satisfies the same small deviation principle as a one-dimensional standard Brownian motion. As far as Chung's LIL for $X_{t}$ is concerned, the first component dominates when $t \rightarrow 0$ with rate $a=\frac{1}{2}$, and the $n$-th component dominates as $t \rightarrow \infty$ with rate $a=\frac{2 n-1}{2}$.

\section{Proofs}

Proof of Theorem 2.6. Let us first prove the small deviation principle (2.5). One has that $\mathbb{P}\left(X_{T}^{*}<\varepsilon\right) \leqslant \mathbb{P}\left(b_{T}^{*}<\varepsilon\right)$, and hence by (2.1) it follows that

$$
\lambda_{1}^{(1)} T \leqslant \liminf _{\varepsilon \rightarrow 0}-\varepsilon^{2} \mathbb{P}\left(X_{T}^{*}<\varepsilon\right) .
$$

Let us now show that

$$
\limsup _{\varepsilon \rightarrow 0}-\varepsilon^{2} \mathbb{P}\left(X_{T}^{*}<\varepsilon\right) \leqslant \lambda_{1}^{(1)} T .
$$

For any $x_{1}, \ldots, x_{n} \in(0,1)$ such that $x_{1}+\cdots+x_{n}=1$ we have that

$$
\begin{aligned}
& \mathbb{P}\left(X_{T}^{*}<\varepsilon\right) \geqslant \mathbb{P}\left(\max _{0 \leqslant t \leqslant T}\left|X_{1}(t)\right|<x_{1} \varepsilon, \ldots, \max _{0 \leqslant t \leqslant T}\left|X_{n}(t)\right|<x_{n} \varepsilon,\right) \\
& \geqslant \mathbb{P}\left(\max _{0 \leqslant t \leqslant T}\left|X_{1}(t)\right|<x_{1} \varepsilon\right) \cdots \mathbb{P}\left(\max _{0 \leqslant t \leqslant T}\left|X_{n}(t)\right|<x_{n} \varepsilon\right),
\end{aligned}
$$


where in the second line we used the Gaussian correlation inequality for the law of the process $\left\{X_{t}\right\}_{0 \leqslant t \leqslant T}$ which is a Gaussian measure on $W_{0}\left(\mathbb{R}^{n}\right)$. Thus,

$$
-\varepsilon^{2} \log \mathbb{P}\left(X_{T}^{*}<\varepsilon\right) \leqslant-\sum_{d=1}^{n} \varepsilon^{2} \log \mathbb{P}\left(\max _{0 \leqslant t \leqslant T}\left|X_{d}(t)\right|<x_{d} \varepsilon\right) .
$$

Note that, for any $d=2, \ldots, n$

$$
\max _{0 \leqslant t \leqslant T}\left|X_{d}(t)\right| \leqslant \int_{0}^{T} \int_{0}^{t_{2}} \cdots \int_{0}^{t_{d-2}} \max _{0 \leqslant t \leqslant T}\left|X_{2}(t)\right| d t_{d-1} \cdots d t_{2}=\frac{T^{d-2}}{(d-2) !} \max _{0 \leqslant t \leqslant T}\left|X_{2}(t)\right|,
$$

and hence

$$
\mathbb{P}\left(\max _{0 \leqslant t \leqslant T}\left|X_{2}(s)\right|<\frac{(d-2) !}{T^{d-2}} x_{d} \varepsilon\right) \leqslant \mathbb{P}\left(\max _{0 \leqslant t \leqslant T}\left|X_{d}(s)\right|<x_{d} \varepsilon\right),
$$

and by [8, Theorem 1.1] we have that, for any $d=2, \ldots, n$

$$
\begin{aligned}
0 & \leqslant \limsup _{\varepsilon \rightarrow 0}-\varepsilon^{2} \log \mathbb{P}\left(\max _{0 \leqslant t \leqslant T}\left|X_{d}(t)\right|<x_{d} \varepsilon\right) \\
& \leqslant \lim _{\varepsilon \rightarrow 0}-\varepsilon^{2} \log \mathbb{P}\left(\max _{0 \leqslant t \leqslant T}\left|X_{2}(t)\right|<\frac{(d-2) !}{T^{d-2}} x_{d} \varepsilon\right) \\
& =\lim _{\varepsilon \rightarrow 0}-\varepsilon^{2} \log \mathbb{P}\left(\max _{0 \leqslant t \leqslant T}\left|\int_{0}^{t} b_{s} d s\right|<\frac{(d-2) !}{T^{d-2}} x_{d} \varepsilon\right)=0 .
\end{aligned}
$$

Thus, by (3.1) and (3.2)

$$
-\varepsilon^{2} \log \mathbb{P}\left(X_{T}^{*}<\varepsilon\right) \leqslant-\varepsilon^{2} \log \mathbb{P}\left(b_{T}^{*}<x_{1} \varepsilon\right)-\sum_{d=2}^{n} \varepsilon^{2} \log \mathbb{P}\left(\max _{0 \leqslant t \leqslant T}\left|X_{2}(t)\right|<\frac{(d-2) !}{T^{d-2}} x_{d} \varepsilon\right),
$$

and by (3.3) and (2.1) it follows that

$$
\limsup _{\varepsilon \rightarrow 0}-\varepsilon^{2} \log \mathbb{P}\left(X_{T}^{*}<\varepsilon\right) \leqslant \frac{\lambda_{1}^{(1)}}{x_{1}^{2}} T .
$$

The result follows by letting $x_{1}$ go to one.

Let us now prove (2.6). By (2.2) and Brownian time inversion one has that

$$
\liminf _{t \rightarrow 0} \sqrt{\frac{\log |\log t|}{t}} \max _{0 \leqslant s \leqslant t}\left|b_{s}\right|=\sqrt{\lambda_{1}} \quad \text { a.s. }
$$

Note that

$$
\begin{aligned}
& \left|b_{s}\right|^{2} \leqslant\left|X_{s}\right|^{2}=\left|b_{s}\right|^{2}+\sum_{d=2}^{n}\left|X_{d}(s)\right|^{2} \\
& \leqslant\left|b_{s}\right|^{2}+\max _{0 \leqslant u \leqslant s}\left|b_{u}\right|^{2} \sum_{d=2}^{n} \frac{s^{2 d-2}}{(d-1) !^{2}}
\end{aligned}
$$

and hence

$$
\begin{aligned}
& \frac{\log |\log t|}{t} \max _{0 \leqslant s \leqslant t}\left|b_{s}\right|^{2} \leqslant \frac{\log |\log t|}{t} \max _{0 \leqslant s \leqslant t}\left|X_{s}\right|^{2} \\
& \leqslant \frac{\log |\log t|}{t} \max _{0 \leqslant s \leqslant t}\left|b_{s}\right|^{2}\left(1+\sum_{d=2}^{n} \frac{t^{2 d-2}}{(d-1) !^{2}},\right)
\end{aligned}
$$


By (3.4) it follows that, for any $d=2, \ldots, n$

$$
\lim _{t \rightarrow 0} t^{2 d-2} \frac{\log |\log t|}{t} \max _{0 \leqslant s \leqslant t}\left|b_{s}\right|^{2}=0 \quad \text { a.s. }
$$

and thus

$$
\liminf _{t \rightarrow 0} \frac{\log |\log t|}{t} \max _{0 \leqslant s \leqslant t}\left|X_{s}\right|^{2}=\liminf _{t \rightarrow 0} \frac{\log |\log t|}{t} \max _{0 \leqslant s \leqslant t}\left|b_{s}\right|^{2}=\lambda_{1} \quad \text { a.s. }
$$

which completes the proof of (2.6). Let us now prove (2.7). Set

$$
\phi(t):=\frac{\log \log t}{t} .
$$

By (2.4) we have that, for any $d=1, \ldots, n-1$

$$
\liminf _{t \rightarrow \infty} \phi(t)^{\frac{2 n-1}{2}} \max _{0 \leqslant s \leqslant t}\left|X_{d}(s)\right|=\liminf _{t \rightarrow \infty} \phi(t)^{n-d} \phi(t)^{\frac{2 d-1}{2}} \max _{0 \leqslant s \leqslant t}\left|X_{d}(s)\right|=0 \text { a.s. }
$$

since $\phi(t) \rightarrow 0$ as $t \rightarrow \infty$. Note that

$$
\left|X_{n}(s)\right|^{2} \leqslant\left|X_{s}\right|^{2}=\sum_{d=1}^{n-1}\left|X_{d}(s)\right|^{2}+\left|X_{n}(s)\right|^{2},
$$

and hence

$$
\begin{aligned}
& \phi(t)^{2 n-1} \max _{0 \leqslant s \leqslant t}\left|X_{n}(s)\right|^{2} \leqslant \phi(t)^{2 n-1} \max _{0 \leqslant s \leqslant t}\left|X_{s}\right|^{2} \\
& \leqslant \sum_{d=1}^{n-1} \phi(t)^{2 n-1} \max _{0 \leqslant s \leqslant t}\left|X_{d}(s)\right|^{2}+\phi(t)^{2 n-1} \max _{0 \leqslant s \leqslant t}\left|X_{n}(s)\right|^{2} .
\end{aligned}
$$

Thus, by (2.4) and (3.5) it follows that

$$
\liminf _{t \rightarrow \infty} \phi(t)^{2 n-1} \max _{0 \leqslant s \leqslant t}\left|X_{s}\right|^{2}=\liminf _{t \rightarrow \infty} \phi(t)^{2 n-1} \max _{0 \leqslant s \leqslant t}\left|X_{n}(s)\right|^{2}=\gamma_{n}^{2} \quad \text { a.s. }
$$

and (2.7) is proven.

\section{References}

[1] Gérard Ben Arous and Jing Wang, Very rare events for diffusion processes in short time, 2019.

[2] Marco Carfagnini, Juraj Földes, and David P. Herzog, A functional law og the iterated logarithm for weakly hypoelliptic diffusions at time zero, 2021.

[3] Marco Carfagnini and Maria Gordina, Small deviations and chung's law of iterated logarithm for a hypoelliptic brownian motion on the heisenberg group, 2020.

[4] Xia Chen and Wenbo V. Li, Quadratic functionals and small ball probabilities for the $m$-fold integrated Brownian motion, Ann. Probab. 31 (2003), no. 2, 1052-1077. MR1964958

[5] Alejandro de Acosta, Small deviations in the functional central limit theorem with applications to functional laws of the iterated logarithm, Ann. Probab. 11 (1983), no. 1, 78-101. MR682802

[6] Lars Hörmander, Hypoelliptic second order differential equations, Acta Math. 119 (1967), 147-171. MR0222474

[7] Nobuyuki Ikeda and Shinzo Watanabe, Stochastic differential equations and diffusion processes, second ed., North-Holland Mathematical Library, vol. 24, North-Holland Publishing Co., Amsterdam, 1989. MRMR1011252

[8] Davar Khoshnevisan and Zhan Shi, Chung's law for integrated Brownian motion, Trans. Amer. Math. Soc. 350 (1998), no. 10, 4253-4264. MR1443196 
[9] A. Kolmogoroff, Über das Gesetz des iterierten Logarithmus, Ann. of Math. 35 (1934), no. 1, 116-117. MR1503147

[10] James Kuelbs and Wenbo V. Li, Metric entropy and the small ball problem for Gaussian measures, J. Funct. Anal. 116 (1993), no. 1, 133-157. MR1237989

[11] James Kuelbs and Wenbo V. Li, Small ball estimates for Brownian motion and the Brownian sheet, J. Theoret. Probab. 6 (1993), no. 3, 547-577. MR1230346

[12] RafałLatał a and Dariusz Matlak, Royen's proof of the Gaussian correlation inequality, Geometric aspects of functional analysis, Lecture Notes in Math., vol. 2169, Springer, Cham, 2017, pp. 265-275. MR3645127

[13] W. V. Li and Q.-M. Shao, Gaussian processes: inequalities, small ball probabilities and applications, Stochastic processes: theory and methods, Handbook of Statist., vol. 19, NorthHolland, Amsterdam, 2001, pp. 533-597. MR1861734

[14] Wenbo V. Li, A Gaussian correlation inequality and its applications to small ball probabilities, Electron. Comm. Probab. 4 (1999), 111-118. MR1741737

[15] Wenbo V. Li and Werner Linde, Approximation, metric entropy and small ball estimates for Gaussian measures, Ann. Probab. 27 (1999), no. 3, 1556-1578. MR1733160

[16] Ditlev Monrad and Holger Rootzén, Small values of Gaussian processes and functional laws of the iterated logarithm, Probab. Theory Related Fields 101 (1995), no. 2, 173-192. MR1318191

[17] Bruno Rémillard, On Chung's law of the iterated logarithm for some stochastic integrals, Ann. Probab. 22 (1994), no. 4, 1794-1802. MR1331204

[18] Thomas Royen, A simple proof of the Gaussian correlation conjecture extended to some multivariate gamma distributions, Far East J. Theor. Stat. 48 (2014), no. 2, 139-145. MR3289621

[19] Qi Man Shao, A note on small ball probability of a Gaussian process with stationary increments, J. Theoret. Probab. 6 (1993), no. 3, 595-602. MR1230348

[20] Michel Talagrand, The small ball problem for the Brownian sheet, Ann. Probab. 22 (1994), no. 3, 1331-1354. MR1303647

Acknowledgments. The author thanks Davar Khoshnevisan and Zhan Shi for suggesting the Gaussian correlation inequality $[18,12]$. 


\section{Electronic Journal of Probability Electronic Communications in Probability}

\section{Advantages of publishing in EJP-ECP}

- Very high standards

- Free for authors, free for readers

- Quick publication (no backlog)

- Secure publication $\left(\mathrm{LOCKSS}^{1}\right)$

- Easy interface (EJMS²)

\section{Economical model of EJP-ECP}

- Non profit, sponsored by $\mathrm{IMS}^{3}, \mathrm{BS}^{4}$, ProjectEuclid ${ }^{5}$

- Purely electronic

\section{Help keep the journal free and vigorous}

- Donate to the IMS open access fund ${ }^{6}$ (click here to donate!)

- Submit your best articles to EJP-ECP

- Choose EJP-ECP over for-profit journals

\footnotetext{
${ }^{1}$ LOCKSS: Lots of Copies Keep Stuff Safe http://www. lockss.org/

${ }^{2}$ EJMS: Electronic Journal Management System http://www.vtex.lt/en/ejms.html

${ }^{3}$ IMS: Institute of Mathematical Statistics http://www.imstat.org/

${ }^{4}$ BS: Bernoulli Society http://www. bernoulli-society.org/

${ }^{5}$ Project Euclid: https://projecteuclid.org/

${ }^{6}$ IMS Open Access Fund: http://www.imstat.org/publications/open.htm
} 